Einführung zum Thema

Onkologe $2021 \cdot 27: 848-851$

https://doi.org/10.1007/s00761-021-00993-w

Angenommen: 10. Juni 2021

(c) Springer Medizin Verlag GmbH, ein Teil von Springer Nature 2021

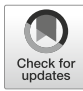

\author{
Martin Reck' - Gunda Leschber ${ }^{2}$ Eleni Gkika ${ }^{3}$ Christiane Bruns ${ }^{4}$ \\ 'LungenClinic Grosshansdorf, Großhansdorf, Deutschland \\ ${ }^{2}$ Evangelische Lungenklinik Berlin, Berlin, Deutschland \\ ${ }^{3}$ Klinik für Strahlenheilkunde, Universitätsklinikum Freiburg, Freiburg, Deutschland \\ ${ }^{4}$ Klinik und Poliklinik für Allgemein-, Viszeral-, Tumor- und Transplantationschirurgie, Uniklinik Köln, Köln, \\ Deutschland
}

\title{
Kleinzelliges Lungenkarzinom
}

Liebe Kolleginnen und Kollegen,

das kleinzellige Lungenkarzinom ist die thorakale Tumorerkrankung, die uns in gleicher Weise Wirksamkeit und Grenzen unserer onkologischen Therapie aufzeigt. Kein anderer Lungentumor spricht so eindrucksvoll auf eine Chemo- oder Strahlentherapie an, aber auch bei keinem anderen Lungentumor kämpfen wir so oft vergeblich gegen das viel zu früh einsetzende Rezidiv.

Während das nichtkleinzellige Lungenkarzinom sich in den letzten Jahren geradezu zu einem Therapiemodell für innovative Therapieansätze jeglicher Art entwickelt hat, stellt sich der Therapiefortschrittbeim kleinzelligen Lungenkarzinom deutlich nüchterner dar - ja, man kann eigentlich sagen, dass wir über Jahrzehnte hinweg trotz erheblicher wissenschaftlicher Anstrengungen keine substanzielle Verbesserung gesehen haben. Das hat sich erfreulicherweise in der letzten Zeit durch neue Therapieansätze und neue Techniken doch geändert, und wir freuen uns, dass wir Ihnen im aktuellen Leitthema zum kleinzelligen Lungenkarzinom einen interdisziplinären Überblick über eine bunte Palette an Innovationen geben dürfen.

$\mathrm{Zu}$ Beginn stellt S. Steurer zusammen mit Kollegen aus dem Universitätsklini-

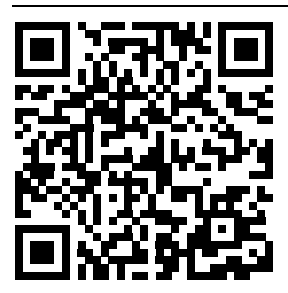

QR-Code scannen \& Beitrag online lesen kum Hamburg-Eppendorf den aktuellen Stand der morphologischen Diagnostik und Differenzialdiagnostik deskleinzelligen Lungenkarzinoms dar. Ein wichtiger innovativer Bereich sind dabei die genetische Struktur und genetische Faktoren des kleinzelligen Lungenkarzinoms, die für die Möglichkeit der zielgerichteten Therapie, aber auch der Immuntherapie von entscheidender Bedeutung sind.

\section{\) Nach Dekaden ohne substanziellen Fortschritt ist die Therapie des kleinzelligen Lungenkarzinoms im Wandel}

Als erster therapeutischer Beitrag folgt von M. Horn u. I. Watermann aus der LungenClinic Grosshansdorf eine Vorstellung der systemtherapeutischen Möglichkeiten beim kleinzelligen Lungenkarzinom. Aufgrund des häufig fortgeschrittenen Tumorstadiums spielt die medikamentöse Behandlung dieses Tumors klinisch eine sehr große Rolle. Ein Schwerpunkt dabei ist die Integration der Immuntherapie in das Therapiekonzept, die nach Jahrzehnten der Stagnation zum ersten Mal zu einer Verbesserung der therapeutischen Effektivität und bei einzelnen Patienten zu einer Verlängerung des Langzeitüberlebens geführt hat. Gleichzeitig zeigen diese Ergebnisse allerdings auch sehr deutlich die Schwierigkeiten einer optimalen Patientenselektion, insbesondere was die Entwicklung spezifischer Biomarker angeht. Ein weiterer Schwerpunkt ist die Frage der zielgerichteten Therapie, die sich allerdings trotz guter prätherapeutischer Konzepte immer noch als sehr schwierig erweist.

Von der Systemtherapie geht es weiter zur Strahlentherapie, der zweiten wichtigen Säule der Behandlung beim kleinzelligen Lungenkarzinom. E. Gkika u. Kolleginnen aus dem Universitätsklinikum Freiburg beschreiben die aktuelle Entwicklung der Strahlentherapie bei Patienten mit nichtmetastasiertem oder metastasiertem kleinzelligem Lungenkarzinom. Neben der Optimierung der multimodalen Therapie im lokal begrenzten Stadium geht es dabei auch um Möglichkeiten der konsolidierenden Strahlentherapie und Optionen einer Bestrahlung des zentralen Nervensystems bei Patienten mit fortgeschrittenen Stadien. Durch die rasante technische Entwicklung der Strahlentherapie in den letzten Jahren ergeben sich hier ganz neue Möglichkeiten.

Das kleinzellige Lungenkarzinom war aufgrund der raschen Tumordynamik traditionell keine Entität, bei der man ein operatives Vorgehen in Betracht zog. Nun zeigen S. von Weihe u. D. Ellebrecht aus der LungenClinic Grosshansdorf auf, dass es durchaus Situationen gibt, in denen Patienten von einem chirurgischen Vorgehen profitieren. Kritisch analysieren sie die publizierten Daten zur chirurgischen Behandlung des kleinzelligen Lungenkarzinoms, um dann unter Nutzung eines intensiven Stagings die Patienten zu definieren, bei denen die Chirurgie Teil eines multimodalen Konzepts sein könnte.

Der nächste Beitrag macht einen kleinen Exkurs zu Tumoren, die man früher auch dem kleinzelligen Lungenkarzinom zugeordnet hat und die mittlerweile eine eigene Entität darstellen: die 
Hier steht eine Anzeige.

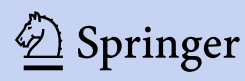


neuroendokrinen Tumoren (NET) der Lunge. F. Weigold u. G. Leschber aus der Evangelischen Lungenklinik in Berlin geben einen Überblick über den aktuellen Stand der Diagnostik und Therapie bei dieser seltenen Tumorerkrankung. Begleitet wird dieser Teil von einer hochinteressanten Darstellung eigener Registerdaten zu Patienten, die mit einem NET an der Lungenklinik behandelt wurden.

M. Sebastian aus der Universitätsklinik Frankfurt und Kollegen schließen dieses Leitthema ab mit einer Analyse der Behandlungsrealität des kleinzelligen Lungenkarzinoms und einer laufenden Registerstudie im Rahmen der CRISPStudie. Wir müssen uns immer klar machen, dass die Mehrzahl aller neuen Therapiedaten an teilweise hoch selektionierten Patienten gewonnen wird - insofern ist dieser Blick in das „alltägliche“ Leben ungemein wertvoll, um die Situation unserer Patienten mit einem kleinzelligen Lungenkarzinom in Deutschland zu verstehen.

Insgesamt sehen wir beim kleinzelligen Lungenkarzinom ähnlich wie bei der nichtkleinzelligen Form mit neuen Diagnostik- und Therapiemöglichkeiten eine deutliche Entwicklung hin zur individualisierten Behandlung unserer Patienten. Immer wichtiger wird gerade bei komplexen, multimodalen Therapieschemata die interdisziplinäre Expertise und Erfahrung spezialisierter Zentren. Nur so wird es uns möglich sein, weiterhin neue Behandlungsansätze und vor allem geeignete Biomarker zu identifizieren, die uns bei dieser immer noch sehr schwierig zu behandelnden Erkrankung weiterhelfen.

Wir wünschen Ihnen viel Freude und hoffentlich viel praktischen Nutzen bei der Lektüre der vielfältigen Beiträge und bedanken uns ganz herzlich bei den CoAutoren für ihr großartiges Engagement!

Mit besten Wünschen!

\section{Martin Reck}

Für die Schriftleiter

Christiane Bruns

Für die Herausgeber

\section{Korrespondenzadresse}

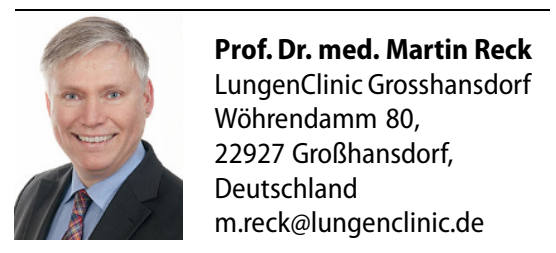

Interessenkonflikt. M. Reck gibt an, Honorare für Beratung und Vorträge von Amgen, AstraZeneca, Boehringer-Ingelheim, BMS, Lilly, Merck, MSD, Mirati, Novartis, Sanofi und Roche erhalten zu haben. G. Leschber, E. Gkika und C. Bruns geben an, dass kein Interessenkonflikt besteht.

\section{Springer Medizin Podcast}

Medizin für Gesundheitsprofis

Der Podcast von SpringerMedizin.de geht seit Juli 2020 spannenden Fragen aus der Welt der Medizin nach immer freitags erweitert eine neue Folge das bereits bestehende OnlineAngebot.

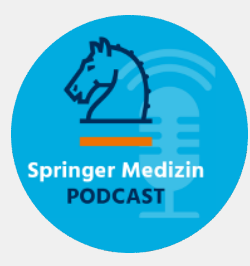

„Mit unserem Podcast möchten wir all jene ansprechen, die sich auf medizinische Themen in einer, gewissen Flughöhe' einlassen möchten", erklärt Dr. Erik Heintz, Chefredakteur von SpringerMedizin.de das neue Format. "Gemeint sind damit Menschen, die sich nicht mit medizinischem Halbwissen zufrieden geben und gerne mehr wissen möchten. Daher legen unsere Redakteurinnen und Redakteure in München und Heidelberg vor allem auf den inhaltlichen Anspruch großen Wert. Unter Bezugnahme auf neue Studien, neue Erkenntnisse und praxisrelevantes Wissen bereiten wir jeden Podcast gründlich vor. Die Themen drehen sich um Gesundheit, Krankheit, Diagnostik, Therapie und Prävention, zu denen wir unsere Fachexpertinnen und Fachexperten aus verschiedenen medizinischen Fachgebieten hinzuziehen.

So entstehen abwechslungsreiche Folgen, die sowohl detailliert Leitlinien abarbeiten, der Evidenz von neuen Studienergebnissen auf den Grund gehen aber auch die Hintergründe zu Erkrankungen und Therapien aufschlüsseln - sodass einerseits die ärztliche Praxis erleichtert und konkrete Tipps für die alltägliche Arbeit vermittelt werden, andererseits aber auch der Horizont über das eigene Fachgebiet hinaus erweitert werden kann.

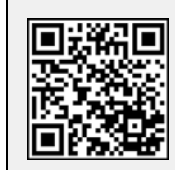

www.springermedizin.de/podcast 
Hier steht eine Anzeige.

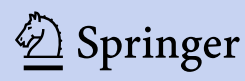

